\title{
Efficient Pheromone Adjustment Techniques in ACO for Ad Hoc Wireless Network
}

\author{
Sharvani G S, \\ $R V$ College of Engineering \\ Bangalore
}

\author{
T.M.Rangaswamy \\ $\mathrm{R} V$ College of Engineering \\ Bangalore
}

\begin{abstract}
Ant Colonies have emergent problem solving nature like food foraging of Ants etc.,. Such problem solving nature of Ant Colonies have inspired emergence of efficient routing algorithms, especially in Ad Hoc Wireless Networks (AWN). They get inspiration from Ants which use simple rules with no direct communication and finds the shortest path by sensing the chemical called 'Pheromone'. Ant Colony Optimization (ACO) is one such routing algorithm. ACO is Agent based routing algorithm and such agent based routing algorithms provide adaptive and efficient utilization of resources in a dynamic environment and also cater for for load balancing and fault management. However there are few issues with ACO to be addressed while adapting ACO for routing on AWN.. One of the Major issues is load balancing due to a problem called 'Stagnation'. Stagnation occurs when all the packets starts travelling on the optimal path and loses packets due to congestion. There are many techniques adopted in ACO to alleviate this problem. This paper focuses on study of different techniques to address the stagnation problem.
\end{abstract}

\section{Keywords}

AdHoc Wireless Netwrok, Swarm Intelligence, Ant Colony Optimization, Stagnation.

\section{INTRODUCTION}

We ask that authors follow some simple guidelines. In essence, we ask you to make your paper look exactly like this document. The easiest way to do this is simply to download the template, and replace the content with your own material

Ad Hoc wireless Networks (AWN) is an autonomous system with no centralized entity controlling the nodes. At the time of communication, nodes rely on many intermediate nodes. Hence the nodes should be more intelligent in the form of a host as well as a router (to forward the nodes). Due to decentralized nature and quick configuration, they are suitable for applications like natural disaster, military. However routing in AWN is a challenging task. There are many dynamic and adaptive protocols developed for routing in AWN. To name a few - Ad Hoc On demand Distance vector protocol (AODV) and Dynamic Source Routing (DSR) [1]. However due to packet and control overhead these protocols were further enhanced to improve the performance.

Swarm Intelligence (SI) is the behavior shown by biological insects such as ants, termites, wasps and bees. These insects independently and collectively exhibit problem solving ability without any centralized control. Each individual follow a set of rules that is influenced by locally available information. This emergent behavior helps these insects to solve any complex problem. Additional properties swarm intelligent systems possess include - robustness against individual misbehavior or loss, the flexibility to change quickly in a dynamic environment, and an inherent parallelism or distributed action [2] [3] [4] [5].

List of general principles in SI routing algorithms, which are derived from Ant Net

- $\quad$ Repeated path sampling

- Stochastic pheromone-based decisions

- Multiple data paths

Repeated path sampling: As compared to traditional distance vector approach, (routing information is calculated based on update messages received from nodes or links) SI routing algorithms gather information through repeated sampling. Due to this real information from multiple ants, which are mutually redundant, avoids the creation of errors in the network.

Stochastic Pheromone based decision: Indirect communication among ants in food foraging by sensing pheromone trail laid by previous ant is stated as "stigmergy" [6] [7] [8]. This is done by ants exploring multiple paths to reach the food source. This is done in a stochastic manner, which helps SI algorithms to be adaptive and handle load.

Multiple Data Paths: To handle resources in network efficiently, an ant carries pheromone table, which is constructed when it traverses from a given source to the destination. To select the best path, it calculates pheromone values in probabilistic manner and takes decision emergently. A pheromone table can have multiple paths to the destination. Whenever there is failure on the optimal path decided by the ant, it checks for next best entry in the pheromone table and follows the path. The typical swarm intelligence system has the following properties:

- It is composed of many individuals called "Agents".

- The individuals are homogeneous

- These agents exploit only local information in the form of "Pheromone" found in the environment and interacts each other indirectly.

- The overall behavior of the system results from the interactions of individuals with each other and with their environment, that is, the group behavior selforganizes. 


\section{BASIC PRINCIPLE OF SWARM- INTELLIGENCE}

Ants that are in search of food, despite a highly changing environment, are able to self-organize based on four principles. They are

- $\quad$ Positive Feed Back

- Negative Feed Back

- Randomness

- Multiple interactions

Positive Feed Back: This improves good solutions by laying more pheromone as more ants travel on that path and tell other ants that it could be the best path to reach the destination.

Negative Feed Back: This mechanism help to destroy bad solution by decaying the pheromone with respect to time. However tuning of pheromone decay is problem specific i.e. good solutions should not be lost fast and bad solutions are evaporated fast.

Randomness: Path chosen by ant is completely random; hence there is possibility of generation of new solutions.

Multiple interactions: In food searching process, food can be found faster if more number of ants interact. Otherwise, with just one ant, the pheromone decays fast and hence many ants are needed to sustain good solution.

Advantages of Swarm Intelligence (SI) in Networks

- Multipath routing - It is possible to generate multiple paths between pair of nodes.

- Efficient route discovery - If optimal path fails, then packets can be easily sent to other neighbors by re-computing next hop probability.

- Fault Tolerant and Distributed - SI algorithm are inherently distributed with no centralized control mechanism. Even if a node or link fails, there will not be heavy loss.

- Scalable - Population of ants are network size specific. The agents may die or reproduce without affecting the performance of the network.

- $\quad$ High Speed - Change in the network is adopted very fats without degrading the performance.

\section{Major categories of SI algorithms Ad Hoc}

\section{wireless networks}

There are two main concepts for Swarm Intelligence Algorithms. They are differentiated clearly based on amount of work published, degree of current activity and its impact on industry especially in the field of computer Science. One such concept called "Ant Colony optimization(ACO)" was inspired by ant species food foraging behavior where as the other concept called "Particle Swarm Optimization" (PSO) was inspired by flocking of birds and school of fishing.

\section{Ant Colony Optimization (ACO)}

ACO was introduced in 1996, which was inspired by an algorithm called "Ant System (AS)" (Dorigo et al 1996). An ant system chooses the best path laid by the previous ant, which went in search of food and has returned. ACO deals with artificial systems, which are inspired from food foraging behavior of real ants, which can find optimal solutions despite changes in the environment. The main idea is indirect communication between the ants by means of pheromone trails, which helps them to find shortest path between their nest and food. ACO are currently applied to applications, such as graph coloring problems, scheduling problems, traveling sales man problem, network routing problem, clustering, robotics etc[2][3].

A major problem with ACO algorithm is "stagnation" (Sim \& Sun, 2003) or premature convergence to local optimum. This occurs when all ants try to follow same path to reach the destination (since there is more pheromone). This when applied in MANETs comes to a convergence state (equilibrium) and attracts all the data packets to follow the same path, which leads to congestion. The next packet without any awareness of the congestion follows a nonoptimal path and loses its packets due to frequent packet drops.

Techniques to alleviate Stagnation in ACO

To mitigate the above problem, different methodologies [9] where adopted and are categorized as follows.

- $\quad$ Privileged Pheromone Laying

- Pheromone-Heuristic Control

- Pheromone Control

In Privileged Pheromone Laying approach, selected subset of ants are used to update pheromone values on the best path. This reduces the probability of ants following the stagnant paths that are non-optimal and congested due to overload.

In the Pheromone-Heuristic Control, ants not only try to find best path based on pheromone concentration on that edge, but also on other factors like queue length, delay and distance. These factors alter the selection of the best path and avoid the stagnation.

Pheromone Control approach is based on controlling the pheromone concentration. Pheromone control can be done in many ways. They are as follows

$$
\begin{aligned}
& \text { 1. Evaporation } \\
& \text { 2. Aging } \\
& \text { 3. Limiting Pheromone } \\
& \text { 4. Smoothing Pheromone }
\end{aligned}
$$

These approaches discourage non-optimal paths and reduce the influence from past experience. In Evaporation approach all the edges with certain pheromone concentration evaporates as the time increases. This is done by an evaporation factor called ' $p$ '. Evaporation not only removes the stale entries, it also balances pheromone concentration in optimal paths. This helps other ants to survey new paths (better). Aging is another technique to reduce stale entries in the network. This approach is based on the fact that older ants deposit lesser pheromone as compared to younger ants. Older ants are those ants that 
have taken longer time to reach the destination. Both Aging and Evaporation aims at finding new best path when there is congestion.

Pheromone can be limited for every edge by placing an upper bound. A variant of the Limiting pheromone is pheromone smoothing in this approach the pheromone is increased along an edge as follows.

$$
\tau_{\mathrm{i}, \mathrm{j}}\left(\mathrm{t}^{\prime}\right)=\tau_{\mathrm{i}, \mathrm{j}}(\mathrm{t})+\delta^{*}\left(\tau_{\max }-\tau_{\mathrm{i}, \mathrm{j}}(\mathrm{t})\right)
$$

Where $\delta$ varies from 0 to 1

It is also observed that smaller amount of pheromone is deposited gradually until the upper bound is reached. Evaporation is done in a uniform manner on all edges. This technique seems to be more effective in avoiding the generation of dominant path [9] [10] [11].

The similarity between Social insects like ants and routing in AWN using agents makes ACO an efficient approach for routing in AWN. The paper attempts to analyze ACO based routing algorithm that have been developed for AWN. This survey aims at creating awareness for the research scholars about the techniques used in ACO to avoid stagnation, highlight its methodology, etc.

\section{A survey on stagnation avoidance algorithms}

- Elitist Ant System (EAS) by Dorigo et al is a variation of $\mathrm{ACO}[12][13]$. Here pheromone deposits are only for the best path found. The major problem with this algorithm is early stagnation.

- Max-Min Ant systems (MMAS) by T. Stutzle, H Hoos [14][15]overcome the early stagnation problem by adding an extra constraint which says that the pheromone is bounded between maximum and minimum pheromone concentration. It will not only avoid early stagnation, it includes less pheromone values as a part of search .However the minimum threshold value set for pheromone concentration caused stagnation since those paths were never preferred for routing(since less pheromone threshold).

- An application of ACO is Scheduling of flexible manufacturing systems by R Kumar et al [16]. Here an effective method was adopted for scheduling of jobs in manufacturing systems, inspired by ACO. The methodology also deals with quick convergence and stagnation avoidance in which parameters of ACO which control the trail and its visibility are fine tuned. Here 'evaporation co-efficient if made either low or high based on the trail concentration.

- MMAS was further improved by Wong and See, the approach adopted was Minimum Pheromone Threshold Strategy (MPTS)[17]. Here the bound between maximum and minimum threshold was fine tuned based on performance of the network. Thus MPTS avoids stagnation problem by avoiding reinitialization of the pheromone trail. With this property MPTS the information lost was avoided, while retaining the good properties of MMAS.
- David C Mathews on Improved Lower Limits for Pheromone Trails in Ant Colony Optimization adopts improved estimates of the lower pheromone value. This helps algorithms like MMAS (sets implicit pheromone trail limit) and Ant Colony Systems (sets explicit pheromone trail limit) to avoid stagnation [18]. This is achieved by adopting explicit or implicit limits on exponential values for stochastic algorithms.

- Laalaoui Y et al in their work stagnation avoidance for scheduling of real-time tasks [19] presents a heuristic approach using ant system. Here a nonpreemptive scheduling approach is adopted for real time tasks. Stagnation problem which was a problem in Ant system was overcome by including Distance function an extra parameter in the transition rule with the pheromone information. This helped in improving the success ratio.

- Application of ACO in TSP was proposed by E Priya Darshini and Gurpreet Kaur. Author proposes an ACO based image edge detection to solve Travelling Sales Man problem [20]. In this the frame work for trail update was developed in order to avoid stagnation. This frame work evaluates each solution against the last ' $\mathrm{N}$ ' solutions globally constructed by ants. As soon as ' $\mathrm{N}$ ' solutions are available, their moving average ' $A$ ' is computed New value of $A$ is compared with old value. If New A is lower than old, the trail level of the last solution's moves is increased, otherwise it is decreased.

- Alaa Alijanaby et al in their work[21] explains in their paper that the technique helps ants to chose best path based on the history of that path i.e based on the pheromone of Ant's own colony and the average os the pheromone of all other colonies. This helps different colonies work with different level of exploration.

- Raka Jovanovic and Milan Tuba in their paper An ACO algorithm with improved pheromone correction strategy for the Minimum Weight Cover Problem (MWCP) explains that stagnation can be avoided by adopting new Hybridization techniques [22]. Corrections to the pheromone trails are performed based on the best found solution. Based on suspicion whether it is good or bad the search proceeds. It decrements the pheromone trails very fast if it finds more suspicious elements.

- Another Hybrid architecture adopted for stagnation avoidance was proposed by Priyanka Sharma and Dr K Kotecha [23]. In their paper optimization in stagnation Avoidance of ACO based routing of Multimedia Traffic over Hybrid MANETs explains that the age of the ant decides whether the trail should be increased or not. After certain age (keeping some threshold) it will not increase the pheromone value. This helps, in providing QoS in MANETs with the help of ACO.

- Another Application of ACO in TSP was proposed by Zar Chi Su Su Hlaing and May Aye Khine in which stagnation problem was overcome by using information entropy [24]. This helped the algorithm to fine tunes its pheromone trails for local search. 


\section{CONCLUSION}

Ad Hoc Wireless Networks consist of nodes which are resource constrained. Designing efficient performing network is a challenging task. SI offers design principles which were inspired by biological insects that will match the constraints of MANETs. In the past decade many routing protocols were developed for MANETs which was inspired by SI based ACO algorithm. However, the stagnation problem makes the load imbalanced. In this paper we have presented a survey of different techniques to alleviate the stagnation problems. These techniques help AWN to perform efficiently and provide Quality of service.

\section{REFERENCES}

[1] C Siva Ram Murthy \& B S Manoj “ Ad Hoc Wireless Networks Architectures and protocols" Pearson Education, 2nd Edition 2005

[2] Bonabeau, E, Dorigo $M$ and Theraulaz G, "Swarm Intelligence from Natural to Artificial Systems", Oxford University Press, 1999.

[3] Bonabeau, E, Dorigo M and Theraulaz G, " Inspiration for optimization from social insect behavior, Vol 406, $39-42,0028-0836,2000$

[4] Di Caro G, Ducatelle F and Gambarella L M, "AntHocNet" Ant-based Hybrid Routing algorithm for MANETs", In : IDSIA-25-04-2004 Technical Report , 112 Dalle Molle Institute for Artificial Intelligence, Switzerland , 2004

[5] Dorigo M, Birattari M and Stuzle T," Ant Colony Optimization. Artificial Ants as a computational Intelligence Technique ", Technical Report, IRDIA, 1$12,1781-3794,2006$

[6] F Neuman D Sudholt, C Witt," Rigorous analyses for the combination of ant colony optimization and local search", ANTS 2008, Proceedings of the 6th International Conference on ACO and Swarm Intelligence, Springer-Verlag, Berlin, pp 132-143

[7] Rajagopalan S and Shen C C, “ANSI:"A swrm Intelligence-based unicast routing protocol for Hybrid AWN,", Journal of System Architecture, Special issues on Nature Inspired Applied Systems, 2007, 485-504

[8] Purkayastha P and Baras J S , " Convergence results for ant routing algorithm via stochastic approximation and optimization, proceedings of the 46th IEE conference on decision and control ,pp 340-345 2007.

[9] De Rango F , Tropea M , Provato A, Sanmaria A F and Marano S , "Minimum Hop Count and Load Balancing Metrics based on Ant Behavior over HAP Mesh", IEEE GLOBECOMM, pp 1-6, New Orleans, 2008.

[10] De Rango F , Tropea M," Energy saving and Load balancing in wireless adhoc networks through ant basaed routing”, SPECTS, Vol 41, 978-1-2-4244-4165-5,2009

[11] Niaz Morshed Cowdhury, Syed Murtoza , Ershadul H. Choudhury " A new adaptive routing approach based on ANT Colony Optimization(ACO) for Ad Hoc Wireless Networks, Proceedings of International workshop on internet and distributed Computing Systems 2008, pp 5156
[12] Ducatelle F, Di Caro G and Gambardella L M, “ Principles and applications of Swarm Intelligence for adaptive routing in telecommunications networks", Swarm Intelligence, 2010.

[13] Dorigo M, V Maniezzo and A Colorni, " The Ant system : Optimization by a Colony of Cooperating Agents.". IEEE Transactions on Systems , Man and cyberneticsPart B, pp 29-41, 1996

[14] T Stutzle, H H Hoos ,"Max-Min Ant system" Future Generation Computing Syst,(2000), PP 889-914.

[15] T Stutzle, H H Hoos, “ Improvements on the ant system: Introducing the max min ant system, in : Third International Conference on Artificial Neural Networks and Genetic Algorithms, Springer Verlag, University of East Anglia, Norwich, 1998, pp 245-249

[16] R Kumar, M K Tiwari and R Shankar, " Scheduling of flexible manufacturing systems: an ant colony optimization approach", Proceedings Instn Mech Engrs Vol 217, Part B: J Engineering Manufacture, 2003,pp $1443-1453$

[17] Kuan Yew Wong, Phen Chiak See, “A New minimum pheromone threshold strategy(MPTS) for Max-min ant system “, Applied Soft computing, Vol 9, 2009, pp 882888

[18] David C Mathew, “ Improved Lower Limits for Pheromone Trails in ACO”, G Rudolf et al(Eds), LNCS 5199, pp 508-517, Springer Verlag, 2008.

[19] Laalaoui Y, Drias H, Bouridah A and Ahmed R B, “ Ant Colony system with stagnation avoidance for the scheduling of real time tasks", Computational Intelligence in scheduling, IEEE symposium, 2009, pp 16.

[20] E Priya Darshini, “ Implementation of ACO algorithm for EDGE detection and Sorting Salesman problem",International Journal of Engineering science and Technology, Vol 2, pp 2304-2315, 2010

[21] Alaa Alijanaby, KU Ruhana Kumahamud, Norita Md Norwawi, "Interacted Multiple Ant

Colonies optimization Frame work: an experimental study of the evaluation and the exploration techniques to control the search stagnation", International Journal of Advancements in computing Technology Vol 2, No 1 , March 2010, pp 78-85

[22] Raka Jovanovic and Milan Tuba, " An ant colony optimization algorithm with improved pheromone correction strategy for the minimum weight vertex cover problem", Elsvier,Applied Soft Computing, PP 53605366,2011

[23] Priyanka Sharma, Dr K Kotecha, “ Optimization in stagnation avoidance of ACO based routing of Multimedia Traffic over Hybrid MANETs", International Journal of computer science and technology, IJCST, Issue 2, ISSN: 2229-4333(print), 0976-8491(online), 2011

[24] Zar Ch Su Su Hlaing, May Aye Lhine, “ An Ant Colony Optimisation Algorithm for solving Traveling Salesman Problem", International Conference on Information Communication and management( IPCSIT), Vol,6, pp $54-59,2011$ 